\title{
Colon Cancer After Acute Diverticulitis Treatment
}

\author{
Kwang Hoon Oh, Koon Hee Han, Eun Jung Kim, Je Hoon Lee, Kyu Un Choi, Myung Sik Han ${ }^{1}$, \\ Jae Hong $\mathrm{Ahn}^{2}$, Gab Jin Cheon \\ Departments of Internal Medicine, ${ }^{1}$ General Surgery, ${ }^{2}$ Diagnostic Radiology, Gangneung Asan Hospital, University of Ulsan College of \\ Medicine, Gangneung, Korea
}

Diverticulitis is the most common clinical complication of diverticular disease, affecting $10-25 \%$ of the patients with diverticula. The prevalences of diverticulitis and colon cancer tend to increase with age and are higher in industrialized countries. Consequently, diverticulitis and colon cancer have been reported to have similar epidemiological characteristics. However, the relationship between these diseases remains controversial, as is the performance of routine colonoscopy after an episode of diverticulitis to exclude colon cancer. Recently, we experienced three cases of colon cancer after treating acute diverticulitis, based on which we suggest the importance of follow-up colonoscopy after acute diverticulitis.

\section{Keywords: Acute diverticulitis; Colon cancer; Colonoscopy}

\section{INTRODUCTION}

A diverticulum is caused by a change in the structure and the resistance of the colonic wall muscular layer as a result of colon movement disorder or lack of dietary fiber, and its incidence tends to increase with age. In Korea, the number of cases of diverticulitis has increased due to the increase in the prevalence of diverticulosis due to the higher average age of the population and the westernization of eating habits [1]. Because the cause and the tendency of diverticulosis have much in common with those of colon cancer, the causal relationship between the two diseases has been investigated. However, any correlation remains controversial $[2,3]$. Although abdominal computed tomography (CT) is the most accurate method for diagnosing acute diverticulitis, its differentiation from colon cancer in the presence of complications, such as abscess and perforation, is difficult [4]. Thus, whether a follow-up colonoscopy should be performed to detect colon can-

Received: August 16, 2012 • Accepted: March 19, 2013

Correspondence to: Koon Hee Han, M.D.

Department of Internal Medicine, Digestive Disease Center, Gangneung Asan Hospital, University of Ulsan College of Medicine, 38 Bangdong-gil, Gangneung 210-711, Korea

TEL: +82-33-610-4951, Fax: +82-33-641-4960

E-mail: hankoonhee@hanmail.net

(C) 2013 The Korean Society of Coloproctology

This is an open-access article distributed under the terms of the Creative Commons Attribution NonCommercial License (http://creativecommons.org/licenses/by-nc/3.0) which permits unrestricted noncommercial use, distribution, and reproduction in any medium, provided the original work is properly cited. cer after treatment for acute diverticulitis, as well as whether such an investigation should be routine, remains controversial [5].

Here, we report three cases of colon cancer, along with a literature review of patients diagnosed with colon cancer following an initial diagnosis of acute diverticulitis based on abdominal CT findings and clinical opinion. Colon cancer was diagnosed based on a biopsy specimen obtained during either colonoscopy or surgery.

\section{CASE REPORTS}

\section{Case 1}

A 63-year-old female with no specific history visited the emergency room with complaints of dyspepsia and right lower quadrant pain that had begun 5 days prior. Her vital signs were a blood pressure of $111 / 75 \mathrm{mmHg}$, a pulse rate of 100 beats/min, a breathing rate of $24 \mathrm{breaths} / \mathrm{min}$, and a body temperature of $36.3^{\circ} \mathrm{C}$. The patient showed acute illness, and physical findings indicated right lower abdominal tenderness, but there was no rebound tenderness. The peripheral blood examination showed leucocytes had increased to $10,800 / \mathrm{mm}^{3}$ and C-reactive protein (CRP) to 10.7 $\mathrm{mg} / \mathrm{dL}$; liver function tests and electrolytes showed no abnormalities. No specific findings were observed on the plain abdominal $\mathrm{X}$-ray. The abdominal CT findings showed a diverticulum of $\sim 2$ $\mathrm{cm}$ on the outside above the colonic wall of the ileocecal valve and ascending colon, which was accompanied by focal wall thickening, fat infiltration and inflammation of $>5 \mathrm{~cm}$ (Fig. 1A). Based on these findings, the patient was diagnosed with acute diverticu- 


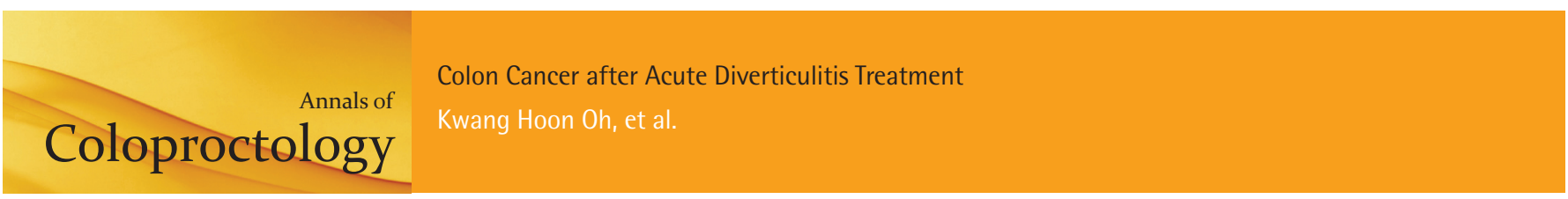

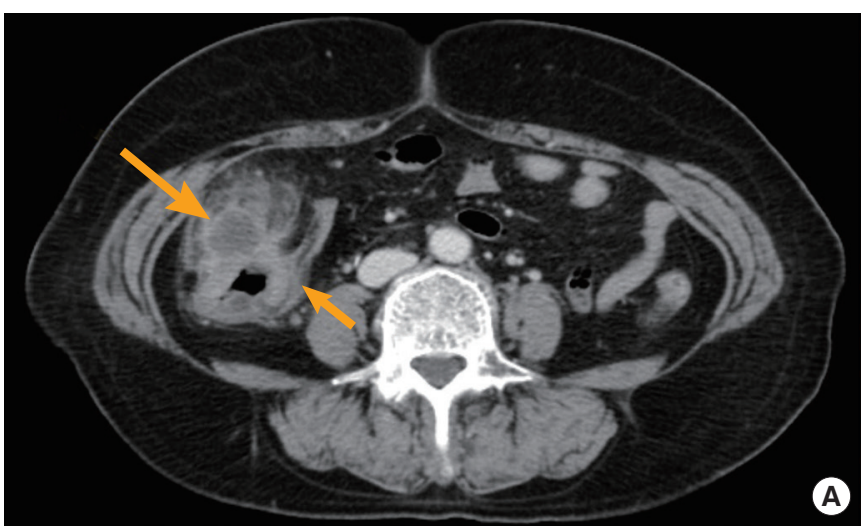
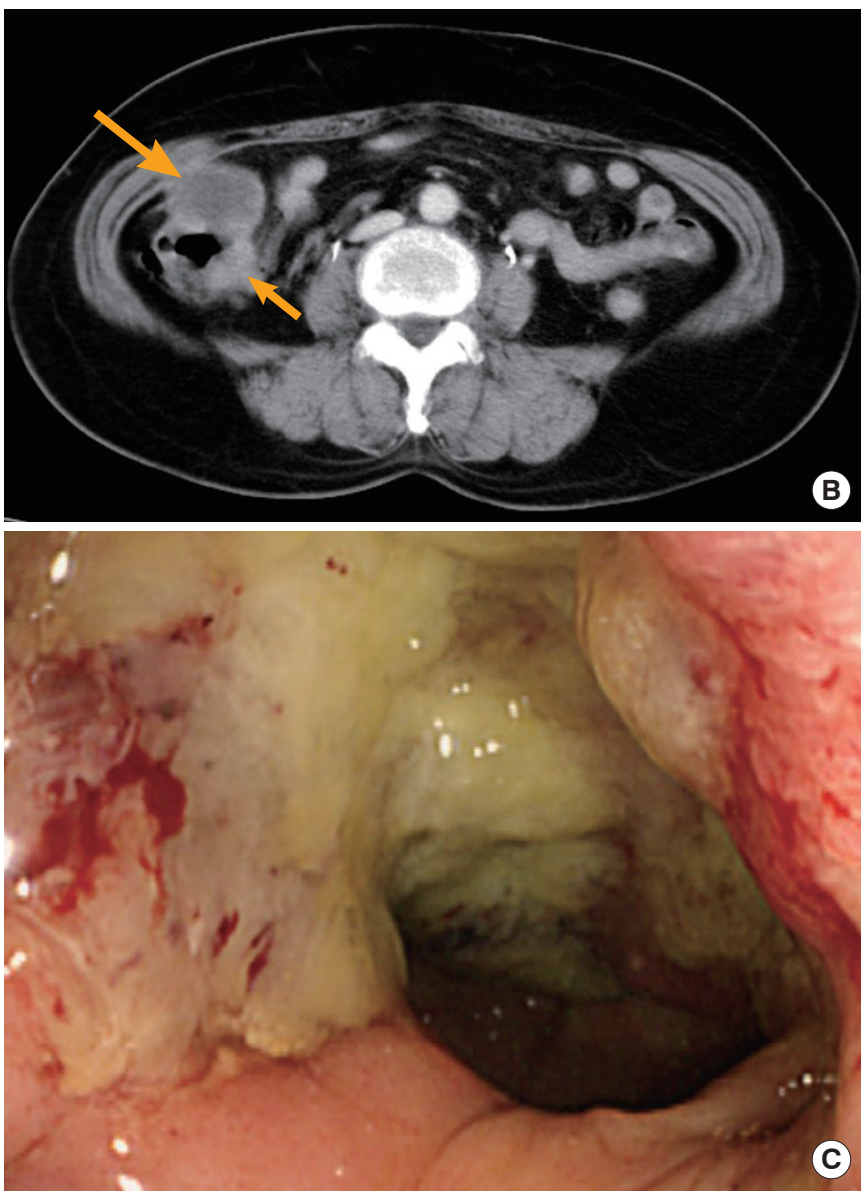

Fig. 1. (A) Axial contrast-enhanced computed tomography (CT) shows a fluid-filled outpouching (arrow) through the ascending colon with pericolic inflammatory changes and focal wall thickening of the ascending colon (short arrow). (B) Axial contrast-enhanced CT performed 3 months later shows improved pericolic infiltration. The cystic lesion in the ascending colon is larger (arrow), and there is persistent focal wall thickening of the ascending colon (short arrow). (C) At colonoscopy, there was an ulcerative lesion from the cecum to the proximal ascending colon, suggesting colon cancer. A dirty exudate covered the lesion, which bled easily on touch. litis and was treated with intravenous antibiotics (ceftriaxone, metronidazole) for 10 days. She was discharged with improved symptoms. One month after discharge, the follow-up CT showed a cystic lesion of $\sim 2.5 \mathrm{~cm}$ remaining in the area of diverticulitis; therefore, an abdominal CT was performed at the follow-up 3 months later to detect abscess formation. The size of the cystic lesion had increased to $\sim 4 \mathrm{~cm}$, and the focal wall thickening remained (Fig. 1B). A biopsy was performed during colonoscopy for differentiation of a chronic abscess, a mucinous cyst and colon cancer. The ulcer lesion was covered with dirty exudates, and bleeding upon easy touch was evident in the proximal ascending colon and ileocecal valve (Fig. 1C). The biopsy results confirmed an adenocarcinoma, moderately differentiated, with a carcinoembryonic antigen (CEA) level of $1.4 \mathrm{ng} / \mathrm{mL}$. A right hemicolectomy was performed, and, based on the surgical specimen, the patient was finally diagnosed with a mucinous adenocarcinoma, moderately differentiated, at the T3N0M0 stage. The patient has been followed up without chemotherapy for 90 months; the cancer has not recurred to date.

\section{Case 2}

A 46-year-old male visited the hospital with the complaint of right lower quadrant pain that had started approximately 1 week prior. At the time of the visit, the patient still had right lower abdominal tenderness and rebound tenderness. The peripheral blood examination showed that the leucocyte level had increased to $12,300 /$ $\mathrm{mm}^{3}$ and the CRP to $2.42 \mathrm{mg} / \mathrm{dL}$, and a plain abdominal X-ray showed no abnormalities. The abdominal CT revealed a diverticulum in the proximal ascending colon and a focal wall thickening of $\sim 6 \mathrm{~cm}$ in the surrounding area, accompanied by peripheral fat infiltration and inflammation, although the appendix was normal. However, lymph nodes around the ileum were enlarged, so colon cancer could not be excluded (Fig. 2A). Clinically, the patient was diagnosed with acute diverticulitis, and the symptoms improved after 5 days of antibiotics (ciprofloxacin) and conservative treatment. One month after discharge, a follow-up colonoscopy was performed for colon cancer differentiation. The colonoscopy showed irregular boundaries surrounding most of the lumen in the proximal ascending colon and an ulceroinfiltrative mass accompanied by changes in the mucosal layer (Fig. 2B). The biopsy results confirmed an adenocarcinoma, poorly differentiated, with signet ring cells and a CEA level of $14.4 \mathrm{ng} / \mathrm{mL}$. The patient underwent a laparotomy, but peritoneal seeding was found in all intraperitoneal areas and was strongly adhered in the ileum. Furthermore, because there was an obstruction in the lesion, only simple bypass surgery (ileum-transverse colon) was performed. Subsequently, the patient received palliative chemotherapy, but died 253 days from the date of surgery.

\section{Case 3}

A 47-year-old male visited the hospital with the complaint of right lower quadrant pain that had started 3 days prior. He had a family 

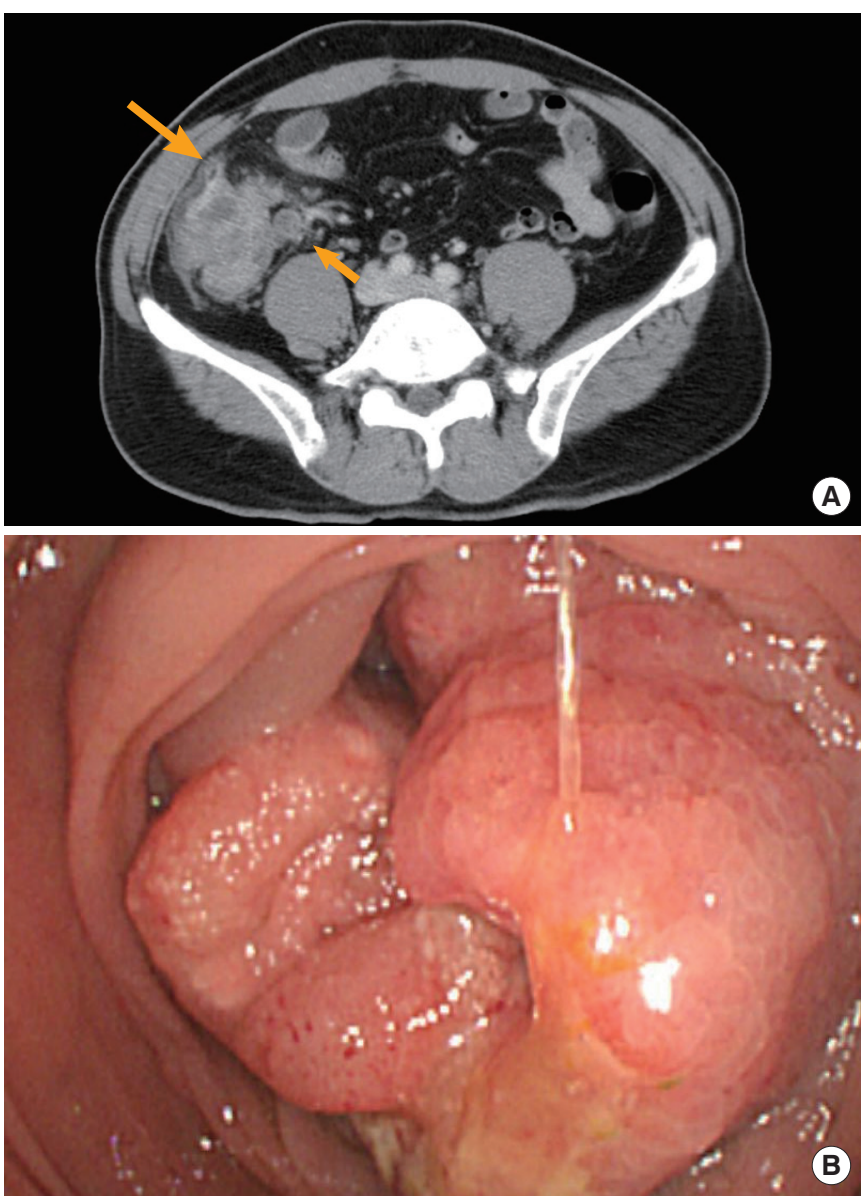

Fig. 2. (A) Axial contrast-enhanced computed tomography showed focal wall thickening of the cecum and proximal ascending colon, with surrounding pericolic fat infiltration and a small diverticulum (arrow). Multiple pericolic lymph nodes were also identified (short arrow). (B) The circular ulcerated infiltrating mass had irregular margins and mucosal changes suggesting colon cancer.

history of his mother dying of colon cancer, but no other medical history. At the time of the visit, the physical examination results showed right lower abdominal tenderness, but no rebound tenderness. The peripheral blood examination showed that leucocytes had increased to $11,900 / \mathrm{mm}^{3}$ and the CRP to $12.31 \mathrm{mg} / \mathrm{dL}$; no specific findings were observed on plain abdominal X-ray. The abdominal CT findings suggested $4 \mathrm{~cm} \times 1 \mathrm{~cm}$ fluid retention in the proximal ascending colon and abscess formation due to diverticulitis perforation towards the outer wall of the ascending colon. In addition, the centripetal focal wall thickening was concurrent; therefore, colon cancer could not be excluded (Fig. 3A). Ultrasound-guided percutaneous abscess drainage was performed, and conservative treatment with intravenous antibiotics (ciprofloxacin, metronidazole) was initiated. Three days later, tubography was performed, and because no residual abscess was found, the patient was discharged seven days after admission. After removal
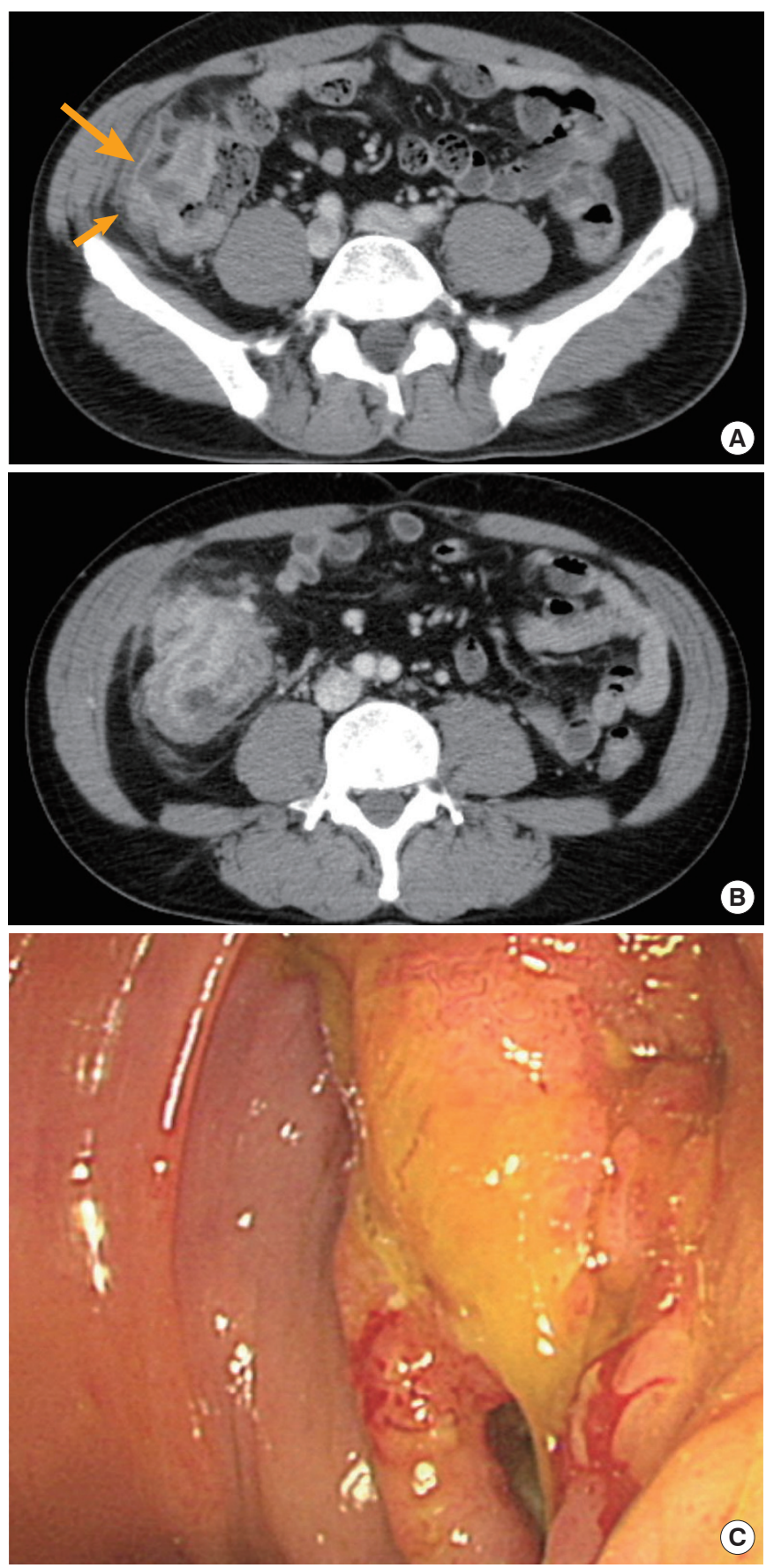

Fig. 3. (A) Axial contrast-enhanced computed tomography (CT) showed an inflamed diverticulum (short arrow), pericolic fluid collection, and pericolic infiltration (arrow). Focal wall thickening of the proximal ascending colon was also seen. (B) Axial contrast-enhanced CT performed 2 years later showed marked circumferential wall thickening of the proximal ascending colon with a thick pericolic infiltration. (C) The edematous inflammatory mass in the proximal ascending colon is suggestive of inflammatory changes due to recurrent diverticulitis or ascending colon cancer. 
of the drainage catheter in the outpatient clinic, a colonoscopy was recommended due to the possibility of colon cancer. However, the patient did not revisit. Approximately two years later, the patient was readmitted with the main complaint of right lower quadrant pain that had started 1 month prior. The abdominal CT suggested diverticulitis-induced bowel perforation in the middle of the ascending colon, accompanied by a thickening of the wall of the ascending colon of $\sim 7$ to $8 \mathrm{~cm}$ in length (Fig. 3B). A colonoscopy was performed to verify the lesion; the results showed an inflammatory mass surrounding the lumen in the proximal ascending colon accompanied by edema (Fig. 3C).

The CEA level was $2.8 \mathrm{ng} / \mathrm{mL}$, and the biopsy result was highgrade dysplasia; however, the abdominal CT and colonoscopy findings suggested colon cancer. Therefore, a right hemicolectomy was performed. Based on the surgical specimen, the patient was finally diagnosed with an adenocarcinoma, moderately differentiated, at the T3N2M0 stage. The patient is receiving palliative chemotherapy and has been followed up for 19 months to date.

\section{DISCUSSION}

The incidences of diverticular disease of the colon and colon cancer in the West are high, but these diseases are relatively rare in the East. However, their incidence is increasing in Korea due to changes in eating habits such as reduction in dietary fiber intake, extended life expectancy, aging of the population, and advances in diagnostic methods such as CT and colonoscopy [6].

Diverticular disease and colon cancer have many epidemiological similarities; therefore, the correlation between these diseases has been investigated. However, any correlation remains controversial. Stefansson et al. $[7,8]$ reported that diverticulitis in the sigmoid colon could increase the occurrence of left colon cancer and proposed a mechanism in which the high concentration of intestinal bacteria in the left colon facilitates the decomposition of feces, resulting in the generation of carcinogenic compounds which are, in turn, trapped inside the diverticulum and constantly irritate the mucous membranes, causing chronic inflammation and cancerous changes. In addition, Kikuchi et al. [9] reported that concurrent chronic inflammation in diverticula could result in cancerous changes and metaplasia, increasing the risk of colon cancer. Meanwhile, some researchers, such as Soran et al. [3] and Loffeld et al. [10], reported that the prevalences of colon cancer and diverticulosis in diverticulitis patients were lower than they were in normal individuals and that no correlation existed between the two diseases [11]. Recent studies showed no significant difference between the prevalence of colon cancer detected during colonoscopy after diverticulitis treatment to the prevalence of colon cancer in ordinary people. For reasons of cost, this has raised questions regarding the need for routine follow-up colonoscopy after treatment of acute diverticulitis $[2,12]$.

Accordingly, controversy regarding the correlation between diverticulitis and colon cancer remains. However, the diverticulitis treatment recommendations published in 2006 by the American Society of Colon and Rectal Surgeons include follow-up colonoscopy for the differentiation of colon cancer, ischemia, and inflammatory bowel diseases after recovery from acute diverticulitis.

Early endoscopy, which is performed during in-patient treatment, has a high probability of perforation, pain and inflammatory stenosis, resulting in a cecum arrival rate as low as $75 \%$ to $82 \%$. Therefore, colonoscopy is recommended approximately six weeks after diverticulitis treatment $[13,14]$. The authors of the present study typically perform colonoscopy six weeks after diverticulitis treatment.

Although abdominal ultrasound with barium enema may be useful for diagnosing acute diverticulitis, abdominal CT has high specificity and sensitivity and a low false-negative rate and so can identify complications with high precision. For this reason, abdominal CT is considered the best method for the diagnosis of diverticulitis $[1,5,12]$. However, in some cases, CT cannot differentiate other abdominal inflammatory diseases, infectious diseases, colon cancer, etc., due to technical error in the shooting, the anatomical state of the contracted colon, and nonspecific concurrent complications in the abdominal cavity, retroperitoneal organs, or abdominal wall [4].

Mesenteric fluid collection and hyperemia of adjacent mesenteric blood vessels indicate diverticulitis when the stenosis transition is gradual and the intestinal wall thickness is $<1 \mathrm{~cm}$. Conversely, lymph node enlargement around the intestine indicates colon cancer. However, when both findings are present, differentiation of diverticulitis and colon cancer is difficult in cases in which cancer infiltration into the intestinal wall is concurrent with fat infiltration; in this situation, biopsy during colonoscopy is necessary $[4,15]$.

These cases are patients who were suspected of having diverticulitis based on CT findings at their initial visit to the hospital and who were discharged with clinical improvement in symptoms af-

Table 1. Clinical features of the patients

\begin{tabular}{lccccccc}
\hline Case & Age/sex & Family history & Site & Complications & $\begin{array}{c}\text { Previous } \\
\text { CFS history }\end{array}$ & $\begin{array}{c}\text { Times of CFS after } \\
\text { diverticulitis treatment (mo) }\end{array}$ \\
\hline 1 & $63 / F$ & None & AC & Abscess & None & 4 & Mucinous adenocarcinoma \\
2 & $46 / M$ & None & AC & None & None & Adenocarcinoma with signet ring cells \\
3 & $47 / M$ & Colon cancer & AC & Perforation abscess & None & 24 \\
\hline
\end{tabular}

CFS, Colonoscopy; AC, ascending colon. 
ter treatment with antibiotics and fluid therapy. The patients were subsequently diagnosed with colon cancer based on the pathology results of specimens obtained during follow-up colonoscopy or surgery. The lesion in all three cases was in the right ascending colon, and there were no findings suggestive of cancer, such as weight loss before admittance, persistent abdominal pain, melena, sudden constipation, etc. Additionally, the patients had no history of undergoing a colonoscopy (Table 1). In case 1 , who was diagnosed with a mucinous adenocarcinoma based on pathologic result, there is a possibility that the diverticulitis and colonic mucinous adenocarcinoma were concurrent at the time of diverticulitis diagnosis; the follow-up CT showed an increase in cystic lesions due to filling of the diverticulum with mucus as a result of the improvement in wall thickening after treatment of inflammation. In case 2, the diverticulitis was concurrent with the focal wall thickening around the diverticulum, peripheral fat infiltration and inflammation. Because of the possibility of concurrent diverticulitis and colon cancer due to the lymph node enlargement around the ileum, a follow-up colonoscopy was performed, and the patient was diagnosed with colon cancer. In case 3 , abscess formation due to diverticulitis perforation was suspected based on CT, but colon cancer could not be excluded because centripetal colonic wall thickening was concurrent. A follow-up colonoscopy was recommended after diverticulitis treatment. However, the patient was not followed up after discharge from the hospital. The colonoscopy was performed two years later, and the patient was diagnosed with cancer at that time. If the colonoscopy had been performed as scheduled after the diverticulitis treatment, the cancer would have been detected earlier and so a better prognosis would have been expected. Although the complications differed, focal colonic wall thickening was concurrent in all three cases based on the abdominal CT findings at the time of diverticulitis diagnosis. Follow-up CT after diverticulitis treatment showed that wall thickening remained or had increased in all three cases in spite of the improvement of inflammation around the diverticulum. Accordingly, although the follow-up CT after diverticulitis treatment showed an improvement in inflammation, if focal wall thickening persists, examination of the lesion by colonoscopy is important for differentiation from colon cancer.

This report evaluated only three patients; therefore, the results should be interpreted with caution. However, follow-up colonoscopy is necessary to observe progress, detect any recurrence, and differentiate diverticulitis from colon cancer after diverticulitis treatment. In particular, colonoscopy is considered necessary if local wall thickening is observed consistently in spite of an improvement in inflammation on the follow-up CT. The importance of follow-up care should be emphasized to patients or guardians both at the time of diagnosis and during diverticulitis treatment.

\section{CONFLICT OF INTEREST}

No potential conflict of interest relevant to this article was re- ported.

\section{REFERENCES}

1. Stollman N, Raskin JB. Diverticular disease of the colon. Lancet 2004;363:631-9.

2. Meurs-Szojda MM, Terhaar sive Droste JS, Kuik DJ, Mulder CJ, Felt-Bersma RJ. Diverticulosis and diverticulitis form no risk for polyps and colorectal neoplasia in 4,241 colonoscopies. Int J Colorectal Dis 2008;23:979-84.

3. Soran A, Harlak A, Wilson JW, Nesbitt L, Lembersky BC, Wienad HS, et al. Diverticular disease in patients with colon cancer: subgroup analysis of national surgical adjuvant breast and bowel project protocol C-06. Clin Colorectal Cancer 2006;6:140-5.

4. Ben Yaacoub I, Boulay-Coletta I, Julles MC, Zins M. CT findings of misleading features of colonic diverticulitis. Insights Imaging 2011;2:69-84.

5. Rafferty J, Shellito P, Hyman NH, Buie WD; Standards Committee of American Society of Colon and Rectal Surgeons. Practice parameters for sigmoid diverticulitis. Dis Colon Rectum 2006;49: 939-44.

6. Moon BC, Kim HS. Developmental pattern and treatment in colon diverticular disease. J Korean Soc Coloproctol 2007;23:30511.

7. Stefansson T, Ekbom A, Sparen P, Pahlman L. Association between sigmoid diverticulitis and left-sided colon cancer: a nested, population-based, case control study. Scand J Gastroenterol 2004; 39:743-7.

8. Stefansson T, Ekbom A, Sparen P, Pahlman L. Increased risk of left sided colon cancer in patients with diverticular disease. Gut 1993;34:499-502.

9. Kikuchi T, Kotanagi H, Kon H, Koyama K, Ito S, Otaka M. Mucosal carcinoma within a colonic diverticulum. J Gastroenterol 1999;34:622-5.

10. Loffeld RJ, Van Der Putten AB. Diverticular disease of the colon and concomitant abnormalities in patients undergoing endoscopic evaluation of the large bowel. Colorectal Dis 2002;4:18992.

11. Krones CJ, Klinge U, Butz N, Junge K, Stumpf M, Rosch R, et al. The rare epidemiologic coincidence of diverticular disease and advanced colonic neoplasia. Int J Colorectal Dis 2006;21:18-24.

12. Westwood DA, Eglinton TW, Frizelle FA. Routine colonoscopy following acute uncomplicated diverticulitis. Br J Surg 2011;98: 1630-4.

13. Almy TP, Howell DA. Medical progress. Diverticular disease of the colon. N Engl J Med 1980;302:324-31.

14. Panish JF. Limitations and complications of colonoscopy. Gastrointest Endosc 1980;26(2 Suppl):20S-21S.

15. Horton KM, Abrams RA, Fishman EK. Spiral CT of colon cancer: imaging features and role in management. Radiographics 2000; 20:419-30. 